Olivares, P.R.; Merellano-Navarro, E.; Perez-Sousa, M.; Collado-Mateo, D. (2021) Fitness, Physical Functionality and Quality of Life in Elderly: A Mediation Analysis. Revista Internacional de Medicina y Ciencias de la Actividad Física y el Deporte vol. 21 (82) pp. 307-318 Http://cdeporte.rediris.es/revista/revista82/artcondicion1250.htm

DOI: https://doi.org/10.15366/rimcafd2021.82.007

\title{
ORIGINAL
}

\section{CONDICIÓN FÍSICA, CAPACIDAD FUNCIONAL Y CALIDAD DE VIDA EN MAYORES: ANÁLISIS DE MEDIACIÓN}

\section{FITNESS, PHYSICAL FUNCTIONALITY AND QUALITY OF LIFE IN ELDERLY: A MEDIATION ANALYSIS}

\author{
Olivares, P.R.'; Merellano-Navarro, E. ${ }^{2}$; Perez-Sousa, M. ${ }^{3}$ y Collado-Mateo, \\ D. ${ }^{5}$ \\ ${ }^{1} \mathrm{PhD}$, Facultad de Educación, Psicología y Ciencias del Deporte, Universidad de Huelva, Huelva \\ (España) Grupo de Investigacion EFISAL. Universidad Autónoma de Chile, Talca (Chile) \\ olivares.pedro@gmail.com \\ 2 PhD, Grupo de Investigacion EFISAL. Universidad Autónoma de Chile, Talca (Chile) \\ emerellanon@uautonoma.cl \\ ${ }^{3} \mathrm{PhD}$, Facultad de Educación, Psicología y Ciencias del Deporte, Universidad de Huelva, Huelva \\ (España) perezsousa@gmail.com \\ $4 \mathrm{PhD}$, Centro de Estudios del Deporte, Universidad Rey Juan Carlos, Madrid (España) \\ danicolladom@gmail.com
}

Financiamiento

El estudio fue apoyado por el Fondo Interno de Investigación de la Universidad Autónoma de Chile (DIUA136-2018).

Código UNESCO / UNESCO Code: 320107 Geriatría / Geriatrics Clasificación Consejo de Europa / Council of Europe Classification: 11 Medicina del Deporte / Sport Medicine

Recibido 31 de mayo de 2019 Received May 31, 2019

Aceptado 28 de marzo de 2020 Accepted March 28, 2020

\section{RESUMEN}

El estudio tiene como objetivo analizar la relación entre la condición física y la calidad de vida relacionada con la salud (CVRS), midiendo el efecto mediador de la funcionalidad física. Se realizaron diferentes pruebas de condición física a adultos mayores. El cuestionario composite physical function se utilizó para medir la funcionalidad física y el EQ-5D-5L para valorar la CVRS. El análisis de mediación confirma el papel de mediador de la funcionalidad física entre la condición física y la CVRS $(B=.051 ; \mathrm{Cl}=.036$ a .067). La asociación directa de 
la condición física y CVRS tuvo un efecto $B=.0314, t=3.313 . p=.001$. Se concluye que la relación entre la condición física y la CVRS está parcialmente mediada por la función física en mayores.

PALABRAS CLAVES: Ancianos, capacidad funcional, Calidad de Vida.

\section{ABSTRACT}

The aim of this work was to analyze the association between fitness and healthrelated quality of life, assessing the physical functionality impact as a mediator. Different tests of physical condition were carried. The composite physical function (CPF) questionnaire was used to measure the physical functionality and the EQ-5D-5L to quantify the HRQoL. The mediation analysis confirms the physical functionality mediator role between fitness and $\mathrm{HRQoL}(\mathrm{B}=.051 ; \mathrm{Cl}=$ .036 to .067). The direct association of fitness and HRQoL had an effect of $\mathrm{B}=.0314, \mathrm{t}=3.313 . \mathrm{p}=.001$. It is concluded that the fitness, regardless its impact on physical functioning, is related to HRQoL.

KEYWORDS: Elderly, functional capacity, quality of life.

\section{INTRODUCCION}

El concepto de calidad de vida relacionada con la salud (CVRS) es ampliamente utilizado en el ámbito científico y está asociada con la manera en como el individuo envejece (Gouveia et al., 2017). Este concepto es multidimensional y se refiere tanto a las condiciones objetivas como a los componentes subjetivos relacionados con la salud de una persona (Urzúa \& Caqueo-Urízar, 2012). Para el adulto mayor, ser funcional significa poder realizar las actividades cotidianas de forma autónoma. La CVRS en este grupo etario, involucra aspectos de salud física, satisfacción de la vida, estado psicológico, bienestar social y la posibilidad de realizar diferentes actividades de la vida diaria de forma autónoma (Herrera \& Guzmán, 2012).

La condición física funcional se define como la capacidad que tiene un individuo de realizar actividades de la vida diaria de forma segura e independiente sin exceso de fatiga (Rikli \& Jones, 1999a). Durante el envejecimiento se produce un descenso en la condición física, especialmente en edades avanzadas (Milanović et al., 2013), principalmente por el declive en los niveles de actividad física, lo cual genera una baja en la resistencia aérobica, flexibilidad, pérdida de fuerza, velocidad, agilidad y equilibrio. Todos estos componentes de la condición física están directamente relacionados a la posibilidad que tiene el adulto mayor de realizar actividades de la vida diaria de forma autónoma (Paterson, Jones, \& Rice, 2007; Paterson \& Warburton, 2010).

En la actualidad, existe estudios que relacionan la condición física con la dependencia funcional en adultos mayores (AM) (Merellano-Navarro, ColladoMateo, García-Rubio, Gusi, \& Olivares, 2017; Rikli \& Jones, 2013; Sardinha, Santos, Marques, \& Mota, 2015). Esta afirmación indica que aquellas personas 
con nivel de condición física en los percentiles más bajos poseen menor nivel de funcionalidad. Por otro lado, se ha demostrado que la condición física se relaciona con la CVRS en población AM (Alves et al., 2019; Chung, Zhao, Liu, \& Quach, 2017; Gusi, Hernandez-Mocholi, \& Olivares, 2015; Horder, Skoog, \& Frandin, 2013; Olivares, Gusi, Prieto, \& Hernandez-Mocholi, 2011; SartorGlittenberg et al., 2014; Wanderley et al., 2011), principalmente, cuando la condición física favorece las dimensiones que se asocian al componente físico de CVRS. Finalmente, existen estudios que relacionan el nivel de dependencia funcional con la CVRS (Boonen et al., 2004; Suriyawongpaisal, Chariyalertsak, \& Wanvarie, 2003), por lo tanto, el nivel de funcionalidad física puede actuar como una variable mediadora al relacionar la condición física y la CVRS.

El análisis de mediación es un procedimiento estadístico utilizado para determinar el vínculo entre dos variables y el grado en que esta relación puede ser modificada, mediada o confundida por una tercera variable (Baron \& Kenny, 1986; Hayes, 2017). El objetivo de este estudio fue analizar la relación entre la condición física y la CVRS, evaluando el efecto de la funcionalidad física como una variable mediadora entre esta relación.

\section{MÉTODO}

\section{PARTICIPANTES}

El estudio se realizó en la Región del Maule (Chile) a través de una medición única. La selección de la muestra fue no probabilística y por conveniencia. Tomando como referencia la última "Encuesta Nacional de Caracterización Socioeconómica (CASEN)" (Ministerio de Desarrollo Social, 2017) la población de adulto mayor de la Región del Maule es de 190931, tomando un margen de error del $5 \%$ y un intervalo de confianza del $95 \%$ se necesitaría una muestra de 384 participantes. La muestra total fue de 406 adultos mayores $\geq 60$ años pertenecientes a distintas ciudades y pueblos de la región del Maule. Se consideraron los siguientes criterios de exclusión: a.- indicar deterioro cognitivo severo en el Test de Pfeiffer (Martínez de la Iglesiaa et al., 2001), b.- problemas motor severo que imposibilitara la realización de los test y c.- hipertensión arterial no controlada. El estudio fue aprobado por el comité de bioética de la Universidad Autónoma de Chile (Ethical Application Ref: $\left.N^{\circ} 028-15\right)$. Los participantes aceptaron los términos del estudio firmando un consentimiento informado.

\section{APLICACIÓN DE CUESTIONARIOS Y TEST DE CONDICIÓN FÍSICA}

La aplicación del cuestionario y la medición de las pruebas de condición física se llevaron a cabo el mismo día. Todos los participantes realizaron un calentamiento antes de las pruebas a través con ejercicios de movilidad articular y estiramiento durante 5 minutos. El tiempo total de aplicación de la prueba fue de aproximadamente 45 minutos. Todas las mediciones fueron realizadas por el equipo de investigación, compuesta por estudiantes de educación física y capacitados por los investigadores con experiencia en estudios en AM. 
Características sociodemográficas y estilos de vida: Se preguntó por la edad, estado civil, nivel educativo, hábitos de tabaco y alcohol y practica de actividad física semanal. La actividad física se categorizó en "0 horas / semana", "3 horas / semana" y " $\geq 3$ horas/ semana", siguiendo estudios previos (Gusi et al., 2012; Olivares et al., 2011).

Calidad de Vida relacionada con la Salud: Para evaluar la CVRS se utilizó la versión en español del EQ-5D-5L (Herdman et al., 2011). Para el cálculo de este instrumento se utilizó los valores del EQ-5D-5L basados en población uruguayos (Augustovski et al., 2016) debido a que entre los disponibles, este es el único desarrollado para un país sudamericano.

Capacidad Funcional: Se midió mediante la escala Composite Physical Function scale (CPF), desarrollado y validado por Rikli and Jones (1998), y adaptada para su uso en Chile (Merellano-Navarro et al., 2015). Para caracterizar a la muestra, los puntajes del CPF fueron categorizados en alta funcionalidad (puntaje 24), moderada funcionalidad (puntaje entre 14-23) y baja funcionalidad (puntaje de 13 o menos) (Rikli \& Jones, 1998).

Condición Física: Para medir la fuerza en las extremidades inferiores se utilizó el Chair stand test (Rikli \& Jones, 1999a). Para la fuerza en las extremidades superiores, se utilizó el test de handgrip (Rodríguez et al., 1998). La flexibilidad de la parte inferior se obtuvo mediante la prueba modificada "Chair Sit-and-Reach test (Jones, Rikli, Max, \& Noffal, 1998); (Jones et al., 1998) y la del tren superior mediante el Back Scratch Test (Rikli \& Jones, 1999a). La Agilidad se midió a través del Test de Time Up and Go (TUG) (Rikli \& Jones, 1999b) y la Resistencia aeróbica fue medida con el test de 6 minutos caminando (Enright et al., 2003). Todos estos test se realizaron entre las 9 y 13 horas, después de un calentamiento de 5 minutos consistente en ejercicios de movilidad articular y estiramientos (Olivares, Hernandez-Mocholi, Merellano-Navarro, Gusi, \& Collado-Mateo, 2019).

Peso y Altura: Estos datos se recopilaron siguiendo las técnicas y protocolos de publicaciones anteriores (Lohman, Roache, \& Martorell, 1992), además, se calculó el índice de masa corporal (BMI). El peso corporal se obtuvo utilizando una báscula (modelo 803 , Seca, Ltd) con una precisión de $0,1 \mathrm{~kg}$, y la altura se midió con un tallímetro (modelo 213, Seca, Ltd) con una precisión de 0,1 cm. Para la caracterización de la muestra, el IMC se clasificó según los criterios de la Organización Mundial de la Salud (World Health Organization, 2000).

\section{ANÁLISIS ESTADÍSTICO}

Las características de la muestra se obtuvieron a través de un análisis descriptivo utilizando medias \pm desviación estándar para las variables continuas y distribución de frecuencia para las variables categóricas. Los z-scores de las pruebas de condición física incluidas en el estudio se calcularon y utilizaron para estimar un valor medio como un índice de condición física global. El sexo fue considerado para el cálculo de las z-scores y se invirtieron los z-scores de la prueba TUG. 
Se realizó un análisis de mediación simple utilizando un análisis de trayectoria de mínimos cuadrados ordinarios para examinar si la asociación entre la condición física y la CVRS estaba mediada por la funcionalidad física, utilizando la macro PROCESS para SPSS (IBM, Chicago, IL, USA) (Hayes, 2017). EI modelo estaba compuesto por la aptitud como variable independiente, la puntuación EQ-5D-5L como variable dependiente y el CPF como mediador. La hipótesis de la mediación se probó utilizando el método bootstrap con corrección de sesgo de 10,000 muestras para calcular un intervalo de confianza de 95\%. La estimación puntual se consideró significativa cuando el intervalo de confianza no contenía ceros.

\section{RESULTADOS}

La Tabla 1 muestra las características de los participantes en función del sexo. Un total de 111 hombres (27.33\%) y 295 mujeres $(72.66 \%)$ con una medida de edad de $72.23 \pm 6.84$ participaron en el estudio. Solo el $20 \%$ es normopeso y el $30,3 \%$ realiza 3 horas o más de actividad física por semana. El CPF muestra que el $59,1 \%$ de los participantes posee moderada o baja funcionalidad. La mayoría de las pruebas de condición física muestran diferencias entre sexo. Las mujeres obtuvieron mejores resultados en todas las pruebas, a excepción de los resultados en extremidades superiores. Los resultados de fuerza tren inferior y de resistencia aeróbica fueron similares para ambos sexos. 
Tabla 1. Características de la muestra en función del sexo

\begin{tabular}{|c|c|c|c|}
\hline & $\begin{array}{l}\text { Todos } \\
(n=406)\end{array}$ & $\begin{array}{c}\text { Hombres } \\
(n=111)\end{array}$ & $\begin{array}{l}\text { Mujeres } \\
(n=295)\end{array}$ \\
\hline Edad (años) & $72.23 \pm 6.842$ & $74.02 \pm 6.71$ & $71.56 \pm 6.78$ \\
\hline \multicolumn{4}{|l|}{ Años rangos. $\mathrm{n}(\%)$} \\
\hline $60-69$ & $153(37.7)$ & $28(25.2)$ & $125(42.4)$ \\
\hline $70-74$ & $119(29.3)$ & $38(34.2)$ & $81(27.5)$ \\
\hline $75-79$ & $61(15)$ & $21(18.9)$ & $40(13.6)$ \\
\hline $80+$ & $73(18)$ & $24(21.6)$ & $49(16.6)$ \\
\hline \multicolumn{4}{|l|}{ IMC } \\
\hline Normopeso & $81(20.0)$ & $29(26.1)$ & $52(17.6)$ \\
\hline Sobrepeso & $168(41.4)$ & $54(48.6)$ & $114(38.6)$ \\
\hline Obeso & $157(38.7)$ & $28(25.2)$ & $129(43.7)$ \\
\hline \multicolumn{4}{|l|}{$\begin{array}{l}\text { Actividad Física por } \\
\text { semana, n (\%) }\end{array}$} \\
\hline 0 horas/semana & $149(36.7)$ & $47(42.3)$ & 102(34.6) \\
\hline$<3$ horas/semana & $134(33.0)$ & $34(30.6)$ & $100(33.9)$ \\
\hline$\geq 3$ horas/semana & $123(30.3)$ & $30(27.0)$ & $93(31.5)$ \\
\hline \multicolumn{4}{|l|}{ CPF, n(\%) } \\
\hline Alta funcionalidad & $166(40.9)$ & $57(51.4)$ & $109(36.9)$ \\
\hline Moderada funcionalidad & $183(45.1)$ & $39(35.1)$ & $144(48.8)$ \\
\hline Baja funcionalidad & $57(14)$ & $15(13.5)$ & $42(14.2)$ \\
\hline EuroQol & $0.8916 \pm 0.1268$ & $0.9037 \pm 0.1405$ & $0.8870 \pm 0.1212$ \\
\hline \multicolumn{4}{|l|}{ Condición Física } \\
\hline $\begin{array}{l}\text { Flexibilidad extremidad } \\
\text { superior }(\mathrm{cm})\end{array}$ & $-20.90 \pm 13.64$ & $-26.68 \pm 14.77$ & $-18.72 \pm 12.53$ \\
\hline $\begin{array}{l}\text { Flexibilidad extremidad } \\
\text { inferior }(\mathrm{cm})\end{array}$ & $-6.70 \pm 10.29$ & $-9.95 \pm 12.16$ & $-5.47 \pm 9.22$ \\
\hline $\begin{array}{l}\text { Fuerza extremidad } \\
\text { superior }(\mathrm{kg})\end{array}$ & $45.49 \pm 15.13$ & $56.60 \pm 18.15$ & $41.31 \pm 11.31$ \\
\hline $\begin{array}{c}\text { Fuerza extremidad } \\
\text { inferior }\end{array}$ & & & \\
\hline $\begin{array}{l}\text { (número de } \\
\text { repeticiones) }\end{array}$ & $11.58 \pm 4.08$ & $11.35 \pm 4.62$ & $11.68 \pm 3.86$ \\
\hline Agilidad (s) & $8.55 \pm 5.68$ & $10.01 \pm 9.41$ & $8.01 \pm 3.21$ \\
\hline $\begin{array}{l}\text { Resistencia aeróbica } \\
(\mathrm{m})\end{array}$ & $395.15 \pm 119.58$ & $388.10 \pm 134.77$ & $397.66 \pm 113.86$ \\
\hline
\end{tabular}

Abreviaciones: IMC: Índice de masa corporal; CPF: composite physical function; Datos presentados en medias \pm Desviación estándar

El análisis de regresión múltiple realizado para evaluar cada componente del modelo de mediación propuesto, mostró que la condición física se asoció positivamente con la CVRS $(B=.082, t=10.442, p<.001)$ así como con la funcionalidad física. $(B=5.210, t=16.476, p<.001)$. demás, los resultados indicaron que el mediador (funcionalidad física) se asoció positivamente con la 
CVRS $(B=.010, t=8.403, p<.001)$. Los resultados del análisis de mediación confirmaron el papel mediador de la funcionalidad física en la relación entre la condición física y la CVRS con un efecto indirecto significativo $(\mathrm{B}=.051 ; \mathrm{Cl}=$ .036 to .067). Además, los resultados indicaron que el impacto directo de la condición física sobre la CVRS sigue siendo significativo $(B=.031, t=3.313, p$ $=.001)$ al controlar la funcionalidad física, sugiriendo así una mediación parcial. La Figura 1 muestra los resultados y la tabla 2 muestra todos los coeficientes para el modelo.

Tabla 2. Modelo de Coeficientes

\begin{tabular}{|c|c|c|c|c|c|c|c|c|}
\hline \multirow[t]{3}{*}{ Antecedentes } & & \multicolumn{7}{|c|}{ Consequent } \\
\hline & & \multicolumn{3}{|c|}{ M (Funcionalidad Física) } & & \multicolumn{3}{|c|}{ Y (CVRS) } \\
\hline & & Coeff. & DE & $p$ & & Coeff. & $\mathrm{DE}$ & $p$ \\
\hline $\begin{array}{l}\text { X (Condición } \\
\text { Física) }\end{array}$ & $a$ & 5.210 & 0.316 & $<.001$ & $c^{\prime}$ & 0.031 & 0.009 & .001 \\
\hline $\begin{array}{c}\text { M } \\
\text { (Funcionalidad } \\
\text { Física) }\end{array}$ & & --- & --- & --- & $\mathrm{b}$ & 0.010 & 0.001 & $<.001$ \\
\hline \multirow[t]{3}{*}{ Constante } & $\mathrm{i}_{1}$ & 19.578 & 0.218 & $<.001$ & $\mathrm{i}_{2}$ & 0.701 & 0.023 & $<.001$ \\
\hline & & \multicolumn{3}{|c|}{$R^{2}=0.411$} & & \multicolumn{3}{|c|}{$R^{2}=0.339$} \\
\hline & & \multicolumn{3}{|c|}{$\begin{array}{c}F(1.389)=271.454 p< \\
.001\end{array}$} & & \multicolumn{3}{|c|}{$F(2.388)=99.578 p<.001$} \\
\hline
\end{tabular}

CVRS: Calidad de Vida relacionada con la salud; DE: Desviación Estándar.

Figura 1. Efecto indirecto de la condición física en la calidad de vida relacionada con la salud a través de la funcionalidad física.

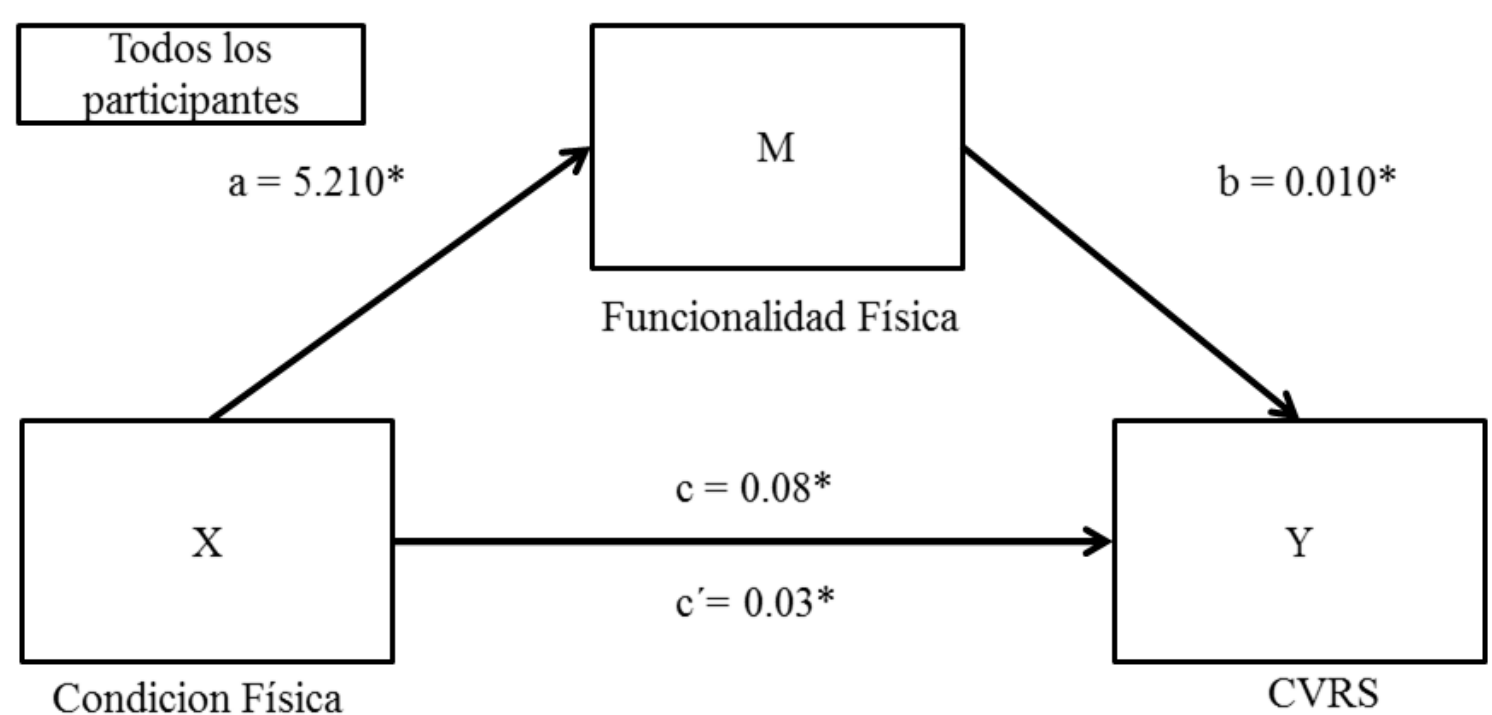

Legend:

Note: ${ }^{*} p<.001$

Indirect effect: $\mathrm{B}=0.051, \mathrm{SE}=0.008,95 \% \mathrm{IC}(0.036-0.067)$ 


\section{DISCUSIÓN}

El concepto de mediación se refiere a la influencia indirecta que una variable independiente tiene sobre una variable dependiente, buscando responder cómo y por qué este efecto se produce a través de una variable mediadora (Hayes, 2017). En el presente estudio, este tipo de análisis nos permitió comprender el impacto de la variable independiente (condición física) sobre la dependiente (CVRS), así como el impacto del mediador de la funcionalidad física en esta asociación. El efecto indirecto obtenido en esta mediación es mayor que el efecto directo de $x-y(\beta=.051$ y $95 \%$ IC .036-.067). Estos resultados muestran la funcionalidad física actúa como mediador parcial en la asociación entre la condición física y la CVRS, y sugieren que el nivel de condición física afecta directamente a la CVRS, como también lo hace indirectamente a través de su impacto en el funcionamiento físico.

Estudios anteriores muestran que un mejor nivel de condición física se asocia con una mejor CVRS (Chung et al., 2017; Gusi et al., 2015; Horder et al., 2013; Jesús Ruiz-Montero, Castillo-Rodríguez, Mikalački, \& Delgado-Fernández, 2015; Olivares et al., 2011), y una mayor independencia funcional. (Merellano-Navarro et al., 2017; Rikli \& Jones, 2013; Sardinha et al., 2015). Según nuestro conocimiento, este es el primer estudio que analiza la asociación de estas tres variables (condición física, funcionalidad física y CVRS) en conjunto, lo que proporciona una mayor comprensión de esta relación.

Los estudios mencionados anteriormente analizaron la asociación entre la condición física y la CVRS utilizando los valores obtenidos en cada prueba de condición física individualmente. En este estudio, las variables de aptitud física se consideraron como el valor promedio estandarizado (Z-score) de los diferentes resultados de las pruebas de condición física de acuerdo con estudios anteriores (Santos et al., 2012). A través de un índice, como el propuesto, se obtiene un valor más representativo de la condición física general de cada persona, lo que permite un análisis más global de la relación entre la condición física y la CVRS. Del mismo modo, la CVRS se evaluó de forma general y no por dimensiones. Se necesitan nuevos estudios para analizar el impacto de la mediación de la funcionalidad física en la condición física, así como las diferentes dimensiones de la CVRS con instrumentos que dan valores de CVRS por dimensiones.

Este estudio tiene algunas limitaciones. El diseño de la sección transversal no nos permitió hacer inferencias de causa e impacto. Además, es plausible que las variables en la Figura 1 estén relacionadas en un orden causal diferente, aunque la secuencia presentada es lógica. Además, una de las dimensiones del EQ-5D está relacionada con la capacidad para realizar las actividades habituales, lo que coincide con el objetivo de evaluación del CPF. Aunque hemos utilizado el EQ5D-5L como puntaje global, en el cual se utilizan las respuestas para todas las dimensiones de este instrumento, esto podría influir en los resultados de la mediación. 


\section{CONCLUSION}

La asociación entre la condición física y la CVRS está mediada parcialmente por la función física en los AM. Esta afirmación sugiere que la condición física, independientemente de su impacto en el funcionamiento físico, está relacionada con la CVRS.

\section{REFERENCIAS}

Alves, W. M., Alves, T. G., Ferreira, R. M., Lima, T. A., Pimentel, C. P., Sousa, E. C., . . A Alves, E. A. (2019). Strength training improves the respiratory muscle strength and quality of life of elderly with Parkinson's disease. The Journal of sports medicine and physical fitness. doi: 10.23736/s00224707.19.09509-4

Augustovski, F., Rey-Ares, L., Irazola, V., Garay, O. U., Gianneo, O., Fernandez, G., . . . Ramos-Goni, J. M. (2016). An EQ-5D-5L value set based on Uruguayan population preferences. Quality of life research : an international journal of quality of life aspects of treatment, care and rehabilitation, 25(2), 323-333. doi: 10.1007/s11136-015-1086-4

Baron, R. M., \& Kenny, D. A. (1986). The moderator-mediator variable distinction in social psychological research: conceptual, strategic, and statistical considerations. Journal of personality and social psychology, 51(6), 11731182. doi: 10.1037//0022-3514.51.6.1173

Boonen, S., Autier, P., Barette, M., Vanderschueren, D., Lips, P., \& Haentjens, P. (2004). Functional outcome and quality of life following hip fracture in elderly women: a prospective controlled study. Osteoporosis international, 15(2), 87-94. doi: 10.1007/s00198-003-1515-z

Chung, P. K., Zhao, Y., Liu, J. D., \& Quach, B. (2017). A canonical correlation analysis on the relationship between functional fitness and health-related quality of life in older adults. Archives of gerontology and geriatrics, 68, 4448. doi: 10.1016/j.archger.2016.08.007

Enright, P. L., McBurnie, M. A., Bittner, V., Tracy, R. P., McNamara, R., Arnold, A., \& Newman, A. B. (2003). The 6-min walk test: a quick measure of functional status in elderly adults. Chest, 123(2), 387-398. doi: 10.1378/chest.123.2.387

Gouveia, E. R. Q., Gouveia, B. R., Ihle, A., Kliegel, M., Maia, J. A., SB, I. Badia, \& Freitas, D. L. (2017). Correlates of health-related quality of life in youngold and old-old community-dwelling older adults. Quality of life research : an international journal of quality of life aspects of treatment, care and rehabilitation, 26(6), 1561-1569. doi: 10.1007/s11136-017-1502-z

Gusi, N., Hernandez-Mocholi, M. A., \& Olivares, P. R. (2015). Changes in HRQoL after 12 months of exercise linked to primary care are associated with fitness effects in older adults. European journal of public health, 25(5), 873-879. doi: 10.1093/eurpub/ckv079

Gusi, N., Prieto, J., Olivares, P. R., Delgado, S., Quesada, F., \& Cebrian, C. (2012). Normative fitness performance scores of community-dwelling older adults in Spain. Journal of Aging and Physical Activity, 20(1), 106126. doi: 10.1123/japa.20.1.106 
Hayes, Andrew F. (2017). Introduction to mediation, moderation, and conditional process analysis: A regression-based approach: Guilford Publications.

Herdman, M, Gudex, Claire, Lloyd, A, Janssen, MF, Kind, P, Parkin, D, . . Badia, Xavier. (2011). Development and preliminary testing of the new five-level version of EQ-5D (EQ-5D-5L). Quality of life research, 20(10), 1727-1736. doi: 10.1007/s11136-011-9903-x

Herrera, P Adela;, \& Guzmán, H Andrés; . (2012). Reflexiones sobre calidad de vida, dignidad y envejecimiento. Revista Médica Clínica Las Condes, 23(1), 65-76.

Hörder, H., Skoog, I., \& Frandin, K. (2013). Health-related quality of life in relation to walking habits and fitness: a population-based study of 75-year-olds. Quality of life research : an international journal of quality of life aspects of treatment, care and rehabilitation, 22(6), 1213-1223. doi: 10.1007/s11136012-0267-7

Ruiz-Montero, P., Castillo-Rodríguez, A., Mikalački, M., \& Delgado-Fernández, M. (2015). Physical Fitness Comparison and Quality of Life between Spanish and Serbian Elderly Women through a Physical Fitness Program. Collegium antropologicum, 39(2).

Jones, C. J., Rikli, R. E., Max, J., \& Noffal, G. (1998). The reliability and validity of a chair sit-and-reach test as a measure of hamstring flexibility in older adults. Res $Q$ Exerc Sport, 69(4), 338-343. doi: 10.1080/02701367.1998.10607708

Lohman, TG, Roache, AF, \& Martorell, R. (1992). Anthropometric standardization reference manual. Medicine \& Science in Sports \& Exercise, 24(8), 952.

Martínez de la Iglesiaa, Jorge, Dueñas Herrerob, Rosa, Onís Vilchesa, M Carmen , Aguado Tabernéa, Cristina , Colomerc, Carmen Albert, \& Luque Luquec, Rogelio (2001). Adaptación y validación al castellano del cuestionario de Pfeiffer (SPMSQ) para detectar la existencia de deterioro cognitivo en personas mayores e 65 años. Medicina Clínica, 117(4), 129134. doi: 10.1016/S0025-7753(01)72040-4

Merellano-Navarro, Eugenio, Collado-Mateo, Daniel, García-Rubio, Javier, Gusi, Narcís, \& Olivares, Pedro R. (2017). Criterion-Referenced Fitness Standards associated with maintaining functional capacity in Chilean Older Adults. Rejuvenation Research. doi: 10.1089/rej.2016.1913

Merellano-Navarro, Eugenio, Lapierre, Michelle, García-Rubio, Javier, Gusi, Narcís, Collado-Mateo, Daniel, \& Olivares, Pedro R. (2015). Traducción y adaptación cultural del cuestionario de independencia física Composite Physical Function para su uso en Chile. Revista médica de Chile, 143(10), 1314-1319. doi: 10.4067/S0034-98872015001000010.

Milanović, Zoran, Pantelić, Saša, Trajković, Nebojša, Sporiš, Goran, Kostić, Radmila, \& James, Nic. (2013). Age-related decrease in physical activity and functional fitness among elderly men and women. Clinical interventions in aging, 8, 549.

Ministerio de Desarrollo Social. (2017). Encuesta de Caracterización Socioeconómica (CASEN). Santiago: Ministerio de Desarrollo Social.

Olivares, P. R., Gusi, N., Prieto, J., \& Hernandez-Mocholi, M. A. (2011). Fitness and health-related quality of life dimensions in community-dwelling middle aged and older adults. Health and quality of life outcomes, 9, 117. doi: 10.1186/1477-7525-9-117 
Olivares, P. R., Hernandez-Mocholi, M., Merellano-Navarro, E., Gusi, N., \& Collado-Mateo, D. (2019). Análisis de la edad sobre la fiabilidad de pruebas fitness en mayores/Analysis of Age on Fitness Reliability in Elderly. Revista Internacional de Medicina y Ciencias de la Actividad Física y el Deporte, 19(76), 627-639. doi: 10.15366/rimcafd2019.76.005

Paterson, Donald H, Jones, Gareth R, \& Rice, Charles L. (2007). Ageing and physical activity: evidence to develop exercise recommendations for older adults. Applied Physiology, Nutrition, and Metabolism, 32 (Suppl. 2E), S69-S108. doi: 10.1139/H07-111

Paterson, Donald H, \& Warburton, Darren ER. (2010). Review Physical activity and functional limitations in older adults: a systematic review related to Canada's Physical Activity Guidelines. International Journal of Behavioral Nutrition and Physical Activity, 7(38), 1-22. doi: 10.1186/1479-5868-7-38

Rikli, R., \& Jones, C. (1998). The reliability and validity of a 6 -minute walk test as a measure of physical endurance in older adults. Journal of Aging and Physical Activity, 6(4), 363-375. doi: 10.1123/japa.6.4.363

Rikli, R., \& Jones, C. (1999a). Development and validation of a functional fitness test for community-residing older adults. Journal of aging and physical activity, 7, 129-161. doi: 10.1123/japa.7.2.129

Rikli, R., \& Jones, C. (1999b). Functional fitness normative scores for communityresiding older adults, ages 60-94. Journal of Aging and Physical Activity, 7, 162-181. doi: 10.1123/japa.7.2.162

Rikli, R., \& Jones, C. (2013). Development and validation of criterion-referenced clinically relevant fitness standards for maintaining physical independence in later years. The Gerontologist, 53(2), 255-267. doi: 10.1093/geront/gns071

Rodríguez, F.A., Gusi, N., Valenzuela, A., Nácher, S., Nogués, J., \& Marina, M. (1998). Evaluation of health-related fitness in adults (I): background and protocols of the AFISAL-INEFC battery. Apunts Educación Física $y$ Deportes, 52, 54-75.

Santos, D. A., Silva, A. M., Baptista, F., Santos, R., Vale, S., Mota, J., \& Sardinha, L. B. (2012). Sedentary behavior and physical activity are independently related to functional fitness in older adults. Experimental Gerontoly, 47(12), 908-912. doi: 10.1016/j.exger.2012.07.011

Sardinha, Luís B, Santos, Diana A, Marques, Elisa A, \& Mota, Jorge. (2015). Criterion-referenced fitness standards for predicting physical independence into later life. Experimental gerontology, 61, 142-146. doi: 10.1016/j.exger.2014.12.012

Sartor-Glittenberg, C., Lehmann, S., Okada, M., Rosen, D., Brewer, K., \& Bay, R. C. (2014). Variables explaining health-related quality of life in community-dwelling older adults. Journal of geriatric physical therapy (2001), 37(2), 83-91. doi: 10.1519/JPT.0b013e3182a4791b

Suriyawongpaisal, P., Chariyalertsak, S., \& Wanvarie, S. (2003). Quality of life and functional status of patients with hip fractures in Thailand. The Southeast Asian journal of tropical medicine and public health, 34(2), 427432.

Urzúa, Alfonso, \& Caqueo-Urízar, Alejandra. (2012). Calidad de vida: Una revisión teórica del concepto. Terapia psicológica, 30(1), 61-71. doi: 10.4067/S0718-48082012000100006 
Wanderley, F. A., Silva, G., Marques, E., Oliveira, J., Mota, J., \& Carvalho, J. (2011). Associations between objectively assessed physical activity levels and fitness and self-reported health-related quality of life in communitydwelling older adults. Quality of life research : an international journal of quality of life aspects of treatment, care and rehabilitation, 20(9), 13711378. doi: 10.1007/s11136-011-9875-x

World Health Organization. (2000). Obesity: preventing and managing the global epidemic: World Health Organization.

Número de citas totales / Total references: 37

Número de citas propias de la revista /Journal's own references: 1

Rev.int.med.cienc.act.fís.deporte- vol. X - número X - ISSN: 1577-0354 